\title{
Time-of-Flight Spectroscopy of Muonic Hydrogen Atoms and Molecules ${ }^{\star}$
}

\author{
M. C. Fujiwara ${ }^{1,2}$, A. Adamczak ${ }^{3}$, J. M. Bailey ${ }^{4}$, G. A. Beer ${ }^{5}$, J. L. Beveridge ${ }^{6}$, \\ M. P. Faifman ${ }^{7}$, T. M. Huber ${ }^{8}$, P. Kammel ${ }^{9}$, S. K. Kim ${ }^{10}$, P. E. Knowles ${ }^{11}$, \\ A. R. Kunselman ${ }^{12}$, V. E. Markushin ${ }^{13}$, G. M. Marshall ${ }^{6}$, G. R. Mason ${ }^{5}$, \\ F. Mulhauser ${ }^{11}$, A. Olin $^{6}$, C. Petitjean ${ }^{13}$, T. A. Porcelli ${ }^{14}$, and J. Zmeskal ${ }^{15}$ \\ (TRIUMF Muonic Hydrogen Collaboration) \\ 1 Department of Physics and Astronomy, University of British Columbia, \\ Vancouver, BC, Canada V6T 2A6 \\ 2 Department of Physics, Faculty of Science, University of Tokyo, \\ Hongo, Tokyo 113-0033 Japan \\ 3 Institute of Nuclear Physics, 31-342 Krakow, Poland \\ 4 Chester Technology, Chester CH4 7QH, England, UK \\ 5 Department of Physics and Astronomy, University of Victoria, \\ Victoria, BC, Canada V8W 2Y2 \\ 6 TRIUMF, Vancouver, BC, Canada, V6T 2A3 \\ 7 Russian Research Center, Kurchatov Institute, Moscow 123182, Russia \\ 8 Department of Physics, Gustavus Adolphus College, St. Peter, MN 56082, USA \\ 9 Department of Physics and Lawrence Berkeley National Laboratory, \\ University of California, Berkeley, CA 94720, USA \\ 10 Department of Physics, Jeonbuk National University, \\ Jeonju City 560-756, S. Korea \\ 11 Institute of Physics, University of Fribourg, CH-1700 Fribourg, Switzerland \\ 12 Department of Physics and Astronomy, University of Wyoming, \\ Laramie, WY 82071-3905, USA \\ 13 Paul Scherrer Institute, CH-5232 Villigen, Switzerland \\ 14 Institute for Medium Energy Physics, Austrian Academy of Sciences, \\ A-1090 Vienna, Austria
}

\begin{abstract}
Studies of muonic hydrogen atoms and molecules have been performed traditionally in bulk targets of gas, liquid or solid. At TRIUMF, Canada's meson facility, we have developed a new type of target system using multilayer thin films of solid hydrogen, which provides a beam of muonic hydrogen atoms in vacuum. Using the time-of-flight of the muonic atoms, the energy-dependent information of muonic reactions are obtained in direct manner. We discuss some unique measurements enabled by the new technique, with emphasis on processes relevant to muon catalyzed fusion.
\end{abstract}

\section{Introduction}

Simple atoms such as hydrogen and helium provide effective testing ground for fundamental theories. Studies of simple exotic atoms could add useful information, despite considerable challenges, both experimentally and theoretically [1].

\footnotetext{
* Contribution to Hydrogen Atom II - Precise Physics with Simple Atomic Systems (Springer, Berlin).
} 
In this article, we shall focus on the reactions of muonic hydrogen atoms and molecules, and their energy dependent properties revealed by a newly developed time-of-flight spectroscopy technique [2, 3,.4.,5], with particular emphasis on reactions related to muon catalyzed fusion phenomena.

\section{Muon Catalyzed Fusion}

A muon is a lepton of the second generation with its mass about 207 times heavier than that of electrons, and has a finite lifetime of $2.2 \mu \mathrm{s}$. It is created from the decay of a pion which is obtained, typically, from intermediate energy $\left(E_{k} \geq 500 \mathrm{MeV}\right)$ collisions of a proton beam on a target nucleus.

A negative muon can participate in a variety of atomic and molecular processes. A muonic atom is formed when a muon stops in matter replacing an electron. A muonic atom interacting with ordinary atoms or molecules can form a muonic molecule. The latter in turn can result in fusion reactions between the nuclei if the target consists of hydrogen isotopes, a phenomenon known as muon catalyzed fusion $(\mu \mathrm{CF})$ [6].

A single muon stopped in a target of deuterium-tritium mixture can catalyze more than 100 fusions, but this number is limited by two major bottle-necks. One is the rate at which a muon can go through the catalysis cycle before its decay (cycling rate), and another is a poisoning process called $\mu-\alpha$ sticking in which, with a probability $\omega_{s} \leq 0.01$, the muon gets captured after the fusion reaction to atomic bound states of the fusion product ${ }^{4} \mathrm{He}$, and hence lost from the cycle (see Section 5 ).

The first bottle-neck, cycling rate is determined mainly by the rate of formation of muonic molecule $d \mu t$. In fact, a straightforward mechanism for molecular formation via Auger process:

$$
\mu t+\mathrm{DX} \rightarrow[(d \mu t) x e]^{+}+e
$$

is much too slow, yielding the fusion efficiency of the order of only one fusion per muon. Here, DX refers to either $\mathrm{D}_{2}$, DT or HD molecule and $x$ the nucleus $p, d$ or $t$. A resonant mechanism, however, can give much higher rates when certain conditions are satisfied. In the resonant formation:

$$
\mu t+\mathrm{DX} \rightarrow\left[(d \mu t)_{11} x e e\right]_{\nu K},
$$

the reaction product is a hydrogen-like molecular complex $[(d \mu t) x e e]$ in which $(d \mu t)^{+}$plays a role of one of the nuclei. The process is resonant, because the energy released upon formation of $d \mu t$ plus the $\mu t$ kinetic energy must match the ro-vibrational $(\nu K)$ excitation energy of $[(d \mu t) x e e]$ in the final state. This is only possible due to the existence of a state $(J, v)=(1,1)$ in $d \mu t$ which is bound very loosely (in the muonic scale) with the binding energy smaller than the dissociation energy of deuterium molecules.

Theoretical calculations [7,8, 9, 10, 11 predict strong enhancement of the formation rate $\lambda_{d \mu t}$ at $\mu t$ energy of order $1 \mathrm{eV}$ (Fig 1), but direct experimental 


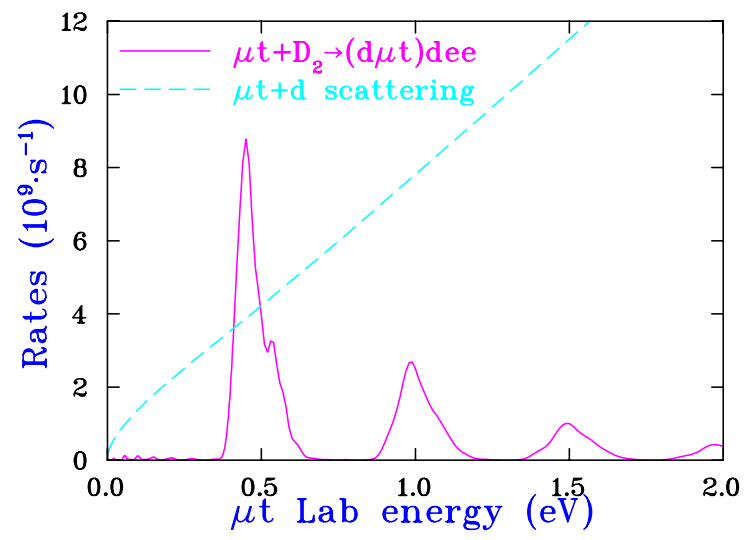

Fig. 1. Resonant molecular formation rate in $\mu t+D_{2}$ collisions calculated for a $3 \mathrm{~K}$ target [7,8,9]. The rates are normalized to the liquid hydrogen density and averaged over $\mu t$ hyperfine states. Also shown is the $\mu t$ elastic scattering rate on the $d$ nucleus [12].

information on such epithermal resonance is scarce, let alone its detailed structure. Traditionally, $\mu \mathrm{CF}$ experiments in general, and molecular formation rate measurements in particular, have been performed in a bulk target made of mixture of hydrogen isotopes, in which complex chains of muon induced reactions take place, making the interpretation difficult and model-dependent. In addition, the resonant energies $\sim 1 \mathrm{eV}$ is difficult to access with the target thermal energy, since it would require a target of several thousand degrees, a formidable task when working with tritium.

At TRIUMF we have developed a new target system which uses multilayer of solid hydrogen thin films [13,14]. The use of $\mu t$ beam obtained from the thin film target provides some unique advantages in tackling the epithermal resonances experimentally: (1) formation process can be isolated, to large extent, from the rest of the cycle, (2) epithermal energies are directly accessible due to the available beam energy, and (3) $\mu t$ time of flight across the drift distance provide information of the resonance energies. Obviously, many technical challenges had to be overcome to make use of the new method.

\section{A beam of muonic hydrogen atoms}

The basic processes involved in creating a beam of muonic tritium atoms 15 can be categorized into four step [16]: atomic formation, acceleration, extraction, and moderation. When a muon is stopped in a thin solid hydrogen target consisting of protium $\left({ }^{1} \mathrm{H}_{2}\right)$ doped with a small amount $\left(c_{t} \sim 0.1 \%\right)$ of tritium, muonic protium atom $(\mu p)$ is mostly formed (atomic formation). The muon quickly transfers from proton to triton [17 to form $\mu t$, the latter more tightly bound due to the reduced mass difference. In the reaction, the $\mu t$ gains about $45 \mathrm{eV}$ of recoil kinetic energy, and is thus accelerated. The $\mu t$ then slow down from the collisions 


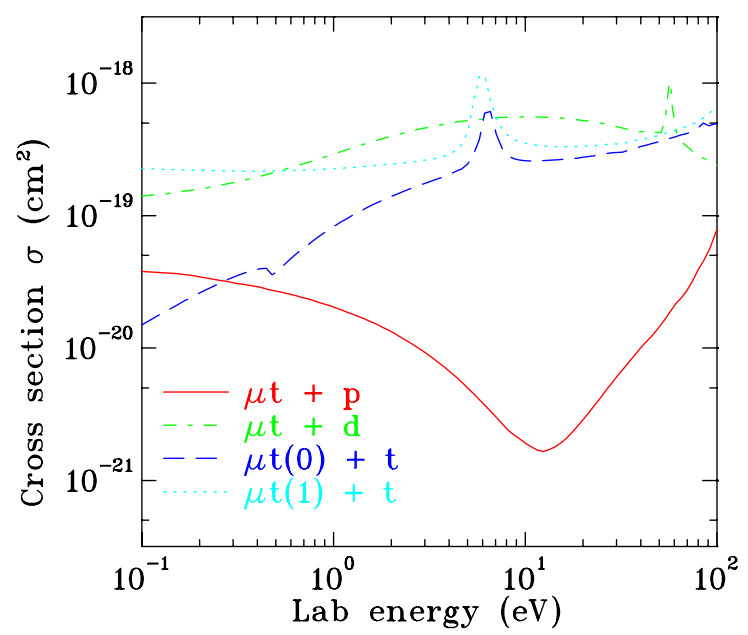

Fig. 2. Scattering cross sections for $\mu t$ with a hydrogen isotope nuclei from Refs. 12, 18, showing the Ramsauer-Townsend minimum at around $10 \mathrm{eV}$ for $\mu t+p \cdot \mu t(F)+t$ cross sections plotted include both elastic and spin exchange reactions, where $\mu t(0)$ is the singlet state and $\mu t(1)$ is the triplet state.

with the rest of the target nuclei (mostly protons), until it reaches about $10 \mathrm{eV}$. At these energies, $\mu t+p$ elastic scattering cross section drops dramatically due to the Ramsauer-Townsend effect, making the rest of the target nearly transparent (Fig 2). The $\mu t$ atom is thus extracted from the layer into vacuum. The energy of the emitted $\mu t$, which is close to the Ramsauer-Townsend minimum of $\sim 10 \mathrm{eV}$ [19], can be controlled to some extent by placing an additional layer, for example of deuterium, on top of the emission layer (moderation). Creation of muonic deuterium $(\mu d)$ is possible in a similar manner with a deuteriumdoped protium target: in fact, the emission of muonic hydrogen atoms was first discovered in this system [21]. See Refs. [22,23, 24] for our measurements involving muonic deuterium. Recently, we also observed emission of muonic protium atoms from pure hydrogen layer [25], though emission mechanism is expected to be completely different 26.

\section{Resonant formation of muonic molecules $d \mu t$}

Figure 3 (a) illustrates the principle of our molecular formation measurements using the atomic beam method [2, 3, 4. 5. A . A beam of $5 \times 10^{3} \mu^{-} / \mathrm{s}$ of momentum $27 \mathrm{MeV} / c$ from the TRIUMF M20B channel was degraded in a $51 \mu \mathrm{m}$ gold target support, and stopped in the upstream (US) emission layer. The $\mu t$ beam, obtained as described above, were slowed via elastic scattering in a $\mathrm{D}_{2}$ moderation layer from some $10 \mathrm{eV}$ to near $1 \mathrm{eV}$ to better match the resonance energies. The $\mu t$, after a few $\mu$ s time of flight (TOF), is collided with a reaction layer in downstream (DS), separated by the drift distance of $17.9 \mathrm{~mm}$ in vacuum. For- 

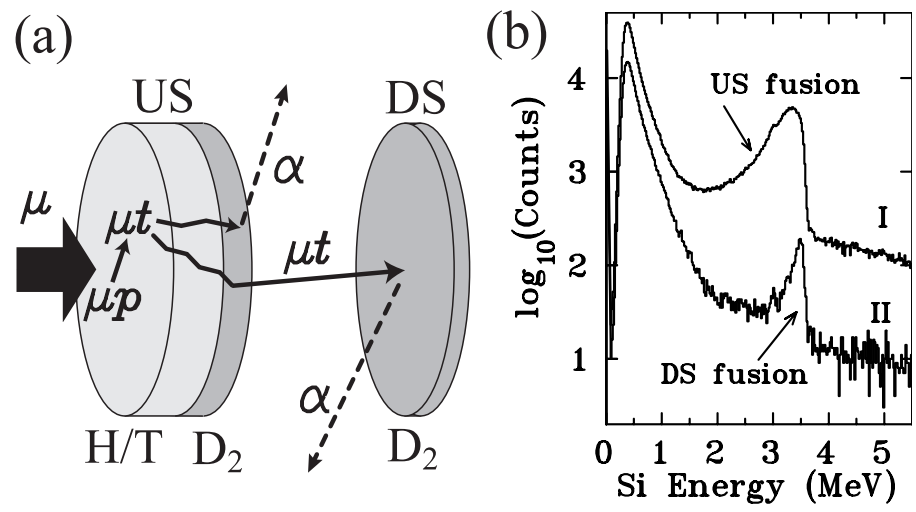

Fig. 3. (a) Schematic of the thin film target for the $d \mu t$ formation measurements consisting of the emission, the moderation and the reaction layers, which are prepared by rapidly freezing hydrogen isotopes on the gold foils (not shown) held in vacuum at 3.5 $\mathrm{K}$ 13]. The layer thickness $\left(3.43 \mathrm{mg} \cdot \mathrm{cm}^{-2}, 96 \mu \mathrm{g} \cdot \mathrm{cm}^{-2}\right.$, and $21 \mu \mathrm{g} \cdot \mathrm{cm}^{-2}$, respectively) were measured off-line via $\alpha$ particle energy loss (see Fig. 5) (b) Measured Si energy spectra with prompt (I: $t>0.02 \mu \mathrm{s}$ ) and delayed (II: $t>1.5 \mu \mathrm{s}$ ) time cuts. Fusion in DS reaction layer is separated from that in US $D_{2}$ due to the $\mu t$ TOF across the vacuum.

mation of $d \mu t$ molecules is detected by observing $3.5 \mathrm{MeV} \alpha$ particles produced in the fusion reaction, $d+t \rightarrow \alpha+n$, which follows the formation. Si detectors placed in the vacuum enables the measurement $\alpha$ with high energy resolution. The background can be determined accurately by "turning off" the DS fusion reactions using the target without the DS layer. This ability to control a specific process, without affecting the rest, is an advantage of the thin film method. (In conventional targets, changing the target conditions would affect many processes simultaneously).

Because the time between muon stop and the fusion $\alpha$ detection (which we call fusion time) is dominated by the $\mu t \mathrm{TOF}$, it provides a measure on molecular formation energy, as long as the energy loss of $\mu t$, due to elastic scattering before the formation in the DS $\mathrm{D}_{2}$, is small. Figure 1 illustrates the simulated correlation between the fusion time and the energy at which molecular formation takes place, when there is little $\mu t$ energy loss. A thin DS layer $(\sim 1 \mu \mathrm{m})$ was thus used in the measurement to minimize the energy loss and maximize the time-energy correlation.

A great deal of efforts were made to understand the systematics of this new method. Fig. 5 and Table 1 give some such examples. The detail of the analysis can be found in Refs. 29. 30]. The resulting DS fusion time spectrum and its comparison with Monte Carlo (MC) simulations are shown in Fig. 6, which clearly establishes the resonance structure. From the time-of-flight analysis of $2036 \pm 116$ DS fusion events, a formation rate consistent with $0.73 \pm(0.16)_{\text {meas }} \pm$ $(0.09)_{\text {model }}$ times the theoretical prediction of Faifman et al. [9] was obtained 


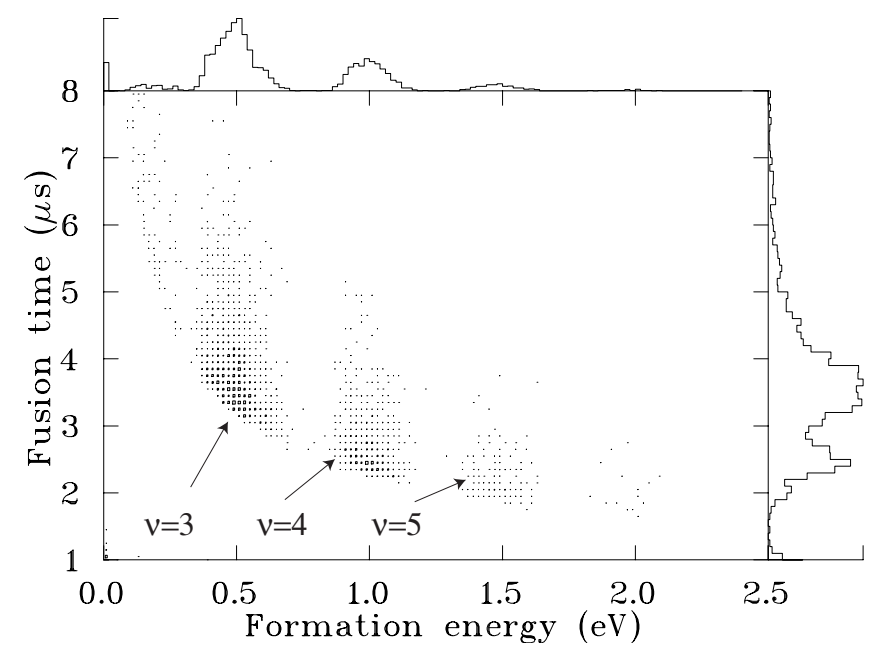

Fig. 4. Simulated correlations between the fusion time and the energy at which molecular formation takes place when there is no $\mu t$ energy loss in DS layer. Corresponding vibrational excitation in the $[(d \mu t) d e e]$ system are also shown.

(the first error refers to the measurement uncertainty including the statistics and the second is that in $\mathrm{MC}$ modeling). The resonance energies were determined from the fit to be $0.940 \pm(0.036)_{\text {meas }} \pm(0.080)_{\text {model }}$ times the theory [9]. Thus, for the first time, the existence of epithermal resonances in $d \mu t$ molecular formation was directly confirmed, and their energies measured. For the largest peak at the resonance energy of $0.423 \pm 0.037 \mathrm{eV}$, our results correspond to the peak rate of $(7.1 \pm 1.8) \times 10^{9} \mathrm{~s}^{-1}$. This is more than an order of magnitude larger than the rates at lower energies, experimentally demonstrating the prospect for high cycling

Table 1. Estimated effects on the formation rate scaling parameter $\mathcal{S}_{\lambda}$ by the systematic uncertainties in the MC modelling.

\begin{tabular}{lr}
\hline \hline MC error source & $\Delta \mathcal{S}_{\lambda} / \mathcal{S}_{\lambda} \%$ \\
\hline$\mu$ beam size & 1.2 \\
Nonuniform $\mu$ stopping (GEANT) & 1.8 \\
$\mu t$ TOF drift distance & 2.6 \\
$\mu p \rightarrow \mu t$ transfer, $p \mu p$ formation & 5.6 \\
$\mu t+d, \mu t+t$ scattering, layer thickness & 5.7 \\
$\mu t+p$ RT minimum energy & 1.1 \\
Resonance Doppler widths in solid & 8.0 \\
Solid state and low energy processes & 5.0 \\
(subthreshold resonances, slow thermalization, & \\
$\mu t$ energy after resonant scattering) & 12.9 \\
\hline Total (in quadrature)
\end{tabular}


$\mu \mathrm{CF}$ in a high temperature target of several thousand degrees. If one assumes the energy levels of the $[(d \mu t) d e e]$ molecule, which have a similar structure to those of ordinary hydrogen, our results imply sensitivity to the binding of energy of the loosely bound $(d \mu t)_{11}$ state with an accuracy comparable to the vacuum polarization and other QED corrections, opening up a new possibility of precision spectroscopy in a quantum few body system.

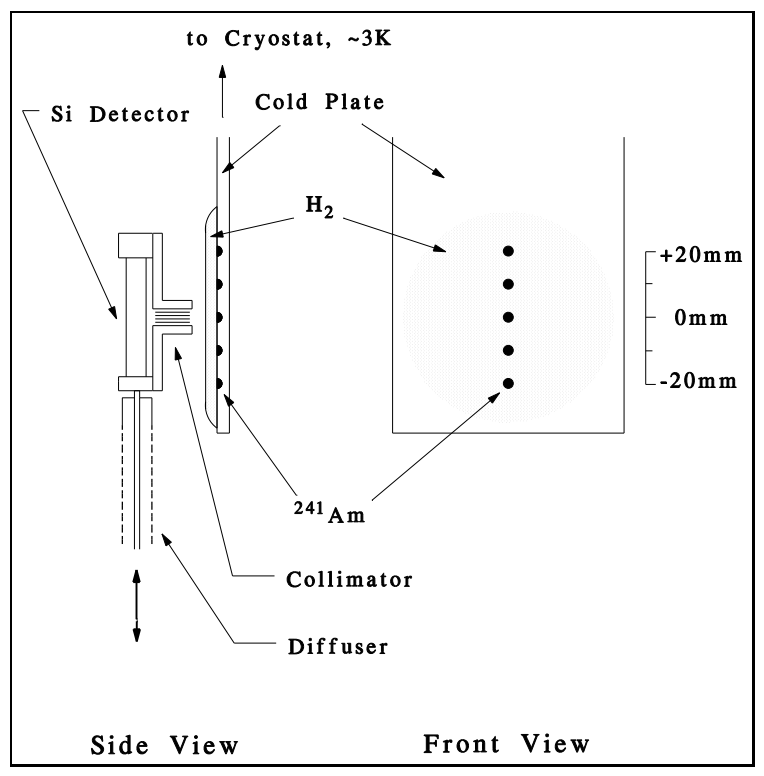

Fig. 5. Calibration measurement of solid hydrogen film layer thickness via energy loss of $\alpha$ particles 27,28. Americium $\alpha$ source is embedded on the surface of the goldplated copper substrate, onto which hydrogen thin film is deposited by releasing the gas through porous sintered metal (diffuser). Silicon detector, mounted on the vertically movable diffuser, measured the $\alpha$ particle energy loss in the film, which is converted the thickness using the stopping power.

Recently $\mu \mathrm{CF}$ using a triple isotope mixture (H/D/T) target has drawn considerable interest 31], particularly because of resonances in the $d \mu t$ formation in the $\mu t+\mathrm{HD}$ collisions, predicted to be even stronger than the $\mu t+\mathrm{D}_{2}$ case. Our thin film target allows us to create $\mu$ t collision with pure HD molecules, and time-of-flight method described here should give a direct test of the theoretical prediction. The data for the $\mu t+\mathrm{HD}$ collision have been collected, and the result will be reported in our future publication 32.

\section{$5 \quad \mu-\alpha$ sticking: yet another bottle-neck}

Although our measurements of epithermal resonant formation indicates molecular formation may no longer be a bottle-neck at appropraite conditions, the 


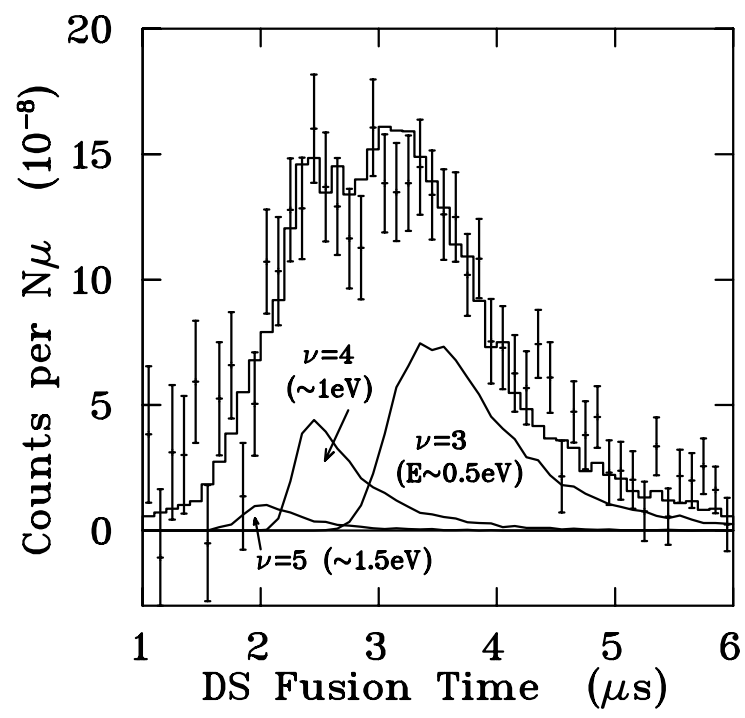

Fig. 6. Time-of-flight fusion spectrum (error bars) and simulation spectrum (histogram), normalized the number of incident muons $N_{\mu}$. Also plotted are simulated contributions from different resonance peaks given by the time-energy correlated events.

process of $\mu-\alpha$ sticking still gives a stringent limit, independent of the cycling rate, on the number of fusions catalyzed by one muon. Intense efforts have been made for nearly two decades to understand this key process, but discrepancies persist between and theory and experiment (including latest PSI [33] and RIKEN-RAL [34] results), the experiment being systematically lower than the theory [35, 36.

Theoretical calculations of sticking are challenging, due to the interplay of the Coulomb and strong interactions in a non-adiabatic few-body system, yet recent predictions, including the effects of nuclear structure and the deviations from the standard sudden approximation, now converge to a few percent [36]. They cannot, however, be readily compared to experiment because most measurements are primarily sensitive to final sticking $\left(\omega_{s}^{\text {fin }}\right)$, which is a combination of initial sticking $\left(\omega_{s}^{0}\right)$, the intrinsic branching ratio for $d \mu t \rightarrow \mu \alpha+n$, and stripping $(R)$, collisional reactivation of the muon from $\mu \alpha$ in the target medium (i.e., $\left.\omega_{s}^{f i n} \equiv \omega_{s}^{0}(1-R)\right)$.

Discrepancies in the experimental sticking results has been in part attributed [33] to the difficulties associated with the conventional neutron method, which include a high model dependence and the need for the absolute neutron calibration. A recent RIKEN-RAL experiment 34] has directly observed, with impressive statistics, X-rays from $\mu \alpha$ deexcitaion following the muon sticking to excited states of $\mu \alpha$, taking advantage of the new intense pulsed muon beam which enabled the large signal enhancement, but unfortunately the determina- 


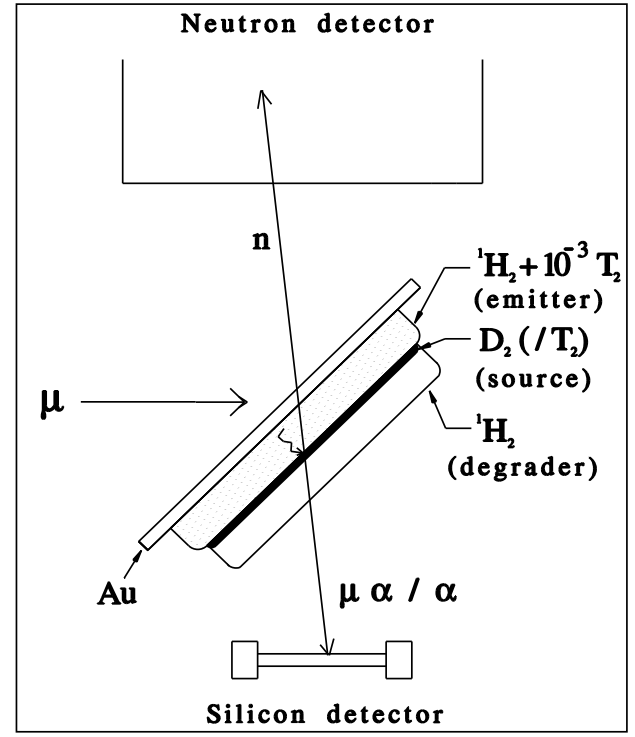

(a) Conceptual view

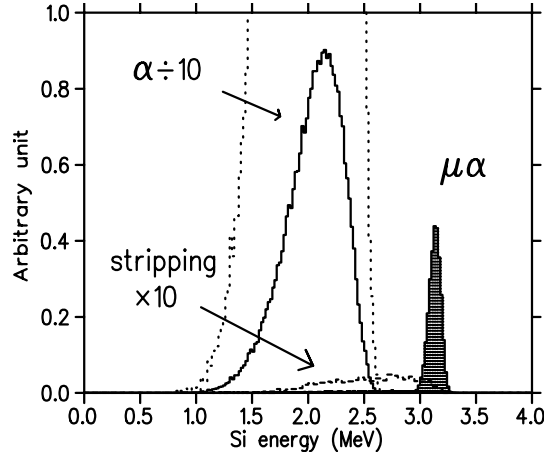

(b) Simulated Si energy

Fig. 7. Proposed direct measurement of $\mu$ - $\alpha$ sticking [37,38]. (a) The $\mu t$ created in the emitter is stopped in the source, where fusion takes place producing $\alpha^{++}$or $(\mu \alpha)^{+}$. The degrader separates the two species by their stopping powers (recall that $d E / d x \propto Z^{2}$ ). Collinear coincidence of the neutron with the charged events suppresses the background. (b) Simulated Si spectrum for the coincidence events, showing a clear separation between $\alpha$ and $\mu \alpha$ peaks.

tion of the sticking probability has to rely on the models of $\mu \alpha$ cascade and stripping, which are yet largely untested.

We have proposed a new direct experiment of sticking using our multi-layer thin film target. The method is illustrated in Fig 0 , and the details are given in Refs. 37, 38. The determination of sticking from the ratio $\mu \alpha /(\mu \alpha+\alpha)$ is simple and model independent. Since the stripping in the degrader is small, the measurement is sensitive to the initial sticking, while the stripping itself process can be systematically studied by varying the degrader thickness. Thus experimental separation of initial sticking and stripping will become possible for the first time.

\section{$6 \quad$ Future prospects}

Currently there is growing interest in the high energy physics community worldwide in developing a muon storage ring for a neutrino factory and/or for a muon collider [39]. Low energy muon science, including muonic atom and molecule 
studies would greatly benefit, if these facilities are realized. Some of the experiments with solid thin films targets are presently limited by the statistics, due to small fraction of muon stops in the target layer, and increased background from muon stopping in the target support and the vacuum chamber [40]. We can expect substantial improvements in the accuracy, as well as entirely new types of experiments with the advent of high intensity muon sources.

\section{Acknowledgements}

This work is supported in part by Canada's NSERC and NRC, USA's DOE and NSF, Swiss National Science Foundation, and NATO Linkage Grant. M.C.F. thanks the support of Rotary Foundation, UBC, Green College, Government of Canada, Westcoast Energy, Nortel and Japan Society of the Promotion of Science.

\section{References}

1. See for example contributions to this volume by T. Yamazaki; K. Jungmann; E. Widmann et al.; M. Hori et al.; and R. Pohl et al.

2. G.M. Marshall et al., Z. Phys. C 56, S44 (1992).

3. G.M. Marshall et al., Hyperfine Interact. 82, 529 (1993).

4. G.M. Marshall et al., ibid. 101/102, 47 (1996).

5. G.M. Marshall et al., ibid. 118, 89 (1999).

6. For reviews see: W. H. Breunlich et al., Ann. Rev. Nucl. Part. Sci. 39, 311 (1989); L. I. Ponomarev, Contemp. Phys. 31, 219 (1990); P. Froelich, Adv. Phys. 41, 405 (1992); J.S. Cohen, in Review of Fundamental Processes and Applications of Atoms and Ions, ed. by C.D. Lin (World Scientific, Singapore, 1993), Ch. 2.

7. M.P. Faifman, L.I. Men'shikov, and T.A. Strizh, Muon Catalyzed Fusion 4, 1 (1989).

8. M.P. Faifman and L.I. Ponomarev, Phys. Lett. 265B, 201 (1991).

9. M.P. Faifman et al., Hyperfine Interact. 101/102, 179 (1996).

10. Yu. V. Petrov et al., Phys. Lett. 331B, 226 (1994).

11. Yu. V. Petrov et al., Phys. Lett. 378B, 1 (1996).

12. C. Chiccoli et al., Muon Catalyzed Fusion, 7, 87 (1992).

13. P.E. Knowles et al., Nucl. Instrum. Methods A 368, 604 (1996).

14. P.E. Knowles et al., Hyperfine Interact. 82, 521 (1993).

15. M.C. Fujiwara et al., Hyperfine Interact. 106, 257 (1997).

16. V.E. Markushin, Hyperfine Interact. 101/102, 155 (1996).

17. F. Mulhauser et al., Phys. Rev. A 53, 3069 (1996).

18. L. Bracci et al., Muon Catalyzed Fusion 4, 247 (1989).

19. M.C. Fujiwara et al., Hyperfine Interact. 118, 151 (1999).

20. F. Mulhauser et al., Hyperfine Interact. 119, 35 (1999).

21. B. Forster et al., Hyperfine Interact. 65, 1007 (1990).

22. R. Jacot-Guillarmod et al., Hyperfine Interact. 101/102, 239 (1996).

23. P. E. Knowles et al., Phys. Rev. A 56, 1970 (1997) [e: 57, 3136 (1998)].

24. A. Olin et al., Hyperfine Interatct. 118, 163 (1999).

25. J. Wozniak et al., Hyperfine Interact. 119, 63 (1999). 
26. A. Adamczak, Hyperfine Interact. 119, 23 (1999).

27. M.C. Fujiwara et al., Nucl. Instrum. Methods A 395, 159 (1997).

28. M.C. Fujiwara et al., Hyperfine Interact. 101/102, 641 (1996).

29. M.C. Fujiwara, Ph.D. thesis, University of British Columbia, Canada (1999).

30. M.C. Fujiwara et al., Phys. Rev. Lett. 851642 (2000).

31. See for example Yu.P. Averin et al., Hyperfine Interact. 118, 121 (1999).

32. T.A. Porcellli et al., to be published.

33. C. Petitjean et al., Hyperfine Interact. 82, 273 (1993).

34. K. Ishida et al., Hyprefine Interact. 118203 (1999).

35. S.S. Gershtein et al., Sov. Phys. JETP 53, 872 (1981); L.N. Bodganova et al., Nucl. Phys. A 454, 653 (1986).

36. C.Y. Hu et al., Phys. Rev. A 49, 4481 (1994); Melezhik, Hyperfine Interact. 101/102, 365 (1996); M. Kamimura et al., 119, 217 (1999).

37. M.C. Fujiwara et al., Hyperfine Interact. 101/102, 613 (1996).

38. M.C. Fujiwara et al., TRIUMF E767 Research Proposal.

39. See for example Proc. of Internationl Workshop of High Intensity Muon Sources, HIMUS-99 (World Scientific, Singapore), to be published.

40. M.C. Fujiwara et al., ibid., to be published. 